\title{
Staging of the Neuroblastomas by Computed Tomography and Magnetic Resonance Imaging
}

\author{
Alexandra Cristina Nicolae, Ioana Gabriela Lupescu
}

Radiology, Medical Imaging and Interventional Radiology Department

Corresponding author:

Fundeni Clinical Institute, Bucharest, Romania

Ioana Gabriela Lupescu, MD

Radiology, Medical Imaging and Interventional Radiology Department Fundeni Clinical Institute, Bucharest 258 Fundeni Str., Sector 2, 022328

E-mail: ilupescu @gmail.com

\section{ABSTRACT}

Neuroblastoma is the most frequent solid extra-cranial malignant tumor in children. A proper staging of the disease allows the selection of the appropriate treatment, including the surgical approach. The present review aims to discuss and illustrate the role of computed tomography (CT) and magnetic resonance (MRI) in the differential diagnosis of the retroperitoneal or mediastinal masses, prior discovered by ultrasound or X-ray examination. Furthermore, it is discussed the role of CT and MRI for staging the patients with a histological proof of neuroblastoma. CT scan is very useful to detect and characterize the thoracic and abdominal tumors and organ metastases, with a special quality in detecting structural microcalcifications. MRI is sensitive for cranio-spinal primary tumors, marrow-bone metastases and mediastinal or pelvic primary or secondary tumors. In conclusion, CT and MRI imaging are mandatory diagnostic tools in staging neuroblastomas, finding the optimal moment for the surgical treatment and establish the risk categories of the children.

Key words: neuroblastoma, staging, computed tomography, magnetic resonance

\section{INTRODUCTION}

Neuroblastoma is a very redoubtable pathology: the most frequent solid extra-cranial malignant tumor in children (1). Neuroblastoma accounts for 10/1 000000 children annually (under 15 years of age) in Europe (2), with approximately 3/100 000 in Romania (3). In the United States 8-10\% of all children's cancers are neuroblastomas (4). The median age at diagnosis is about 2 years (2), with $70 \%$ of the patients discovered in stage 4 disease. Thus, the 5 -year survival rate is only $30 \%$.

A proper diagnosis of neuroblastoma is crucial for primary surgical treatment and precise staging of the disease allows correct inclusion of the patient in one of the multiple treatment choices for neuroblastoma.

The present review aims to discuss and illustrate the role of computed tomography (CT) and magnetic resonance (MRI) in the differential diagnosis of the retroperitoneal or mediastinal masses, prior discovered by ultrasound or 
X-ray examination. Furthermore, it is discussed the role of $\mathrm{CT}$ and MRI for staging the patients with a histological proof of neuroblastoma.

\section{The natural history and clinical course of a neuroblastoma}

The origin of a neuroblastoma can follow tree different pathways:

- most of them arises in the adrenal medulla (medulla glandulae suprarenalis);

- periarterial sympathetic nervous system represented by cervical, abdominal or lateral pelvic (iliac arteries) ganglionar chain;

- ararahidian and presacrat sympathetic system represented by sympathetic chain of the cervical, thoracic, lumbar paravertebral or median pelvic ganglions.

According to these origins, multiple locations of the disease can appear, as follow (2):

I. Abdominal in $60-70 \%$ of cases, from which 32 $35 \%$ originate in adrenal glands;

II. Thoracic (15-20\%);

III. Pelvic (5\%);

IV. Cervical (5\%);

V. Cerebral (1\%);

VI. Unknown (0.5-1\%).

Distant spread can lead to multiple organ metastases which are generally present at the time of diagnosis; their frequencies, attainted at the moment of the diagnosis are, according to some authors (2): sponge bone marrow $(70.8 \%)$, bone $(56 \%)$, ganglionar $(31 \%)$, liver $(30 \%)$, cranio-orbito-epidural (18\%), skin (4\%), pleural (3.5\%), lung (3\%), peritoneal $(2 \%)$, cerebro-meningeal (0.6\%), para-testicular $(0.5 \%)$, ovarian.

Although clinical diagnosis might be difficult and deceiving, there are clinical warning signs, such as: abdominal or limb mass, with or without pain, weight loss, fever, weakness, or, with some specificity - proptosis or the complex of signs named "raccoon eye", "blueberry muffin" syndrome in skin metastases $(6,7)$.

The biological criteria may include increased levels of: serum or urine catecholamines (95\% of patiens), lactate dehydrogenase, ferritin, neuron-specific enolase $(6,7)$.

\section{Staging of neuroblastomas}

Nowadays, neuroblastomas are staged according to the International Neuroblastoma Staging System, as shown in table 1.

All the patients with a histological proof of a neural crest tumor can be classified as follows:

- neuroblastoma or poor stromal neuroblastoma (more than 50\% neuroblasts);

- ganglioneuroblastoma or rich stromal neuroblastoma with more than $50 \%$ stromal tissue (2).

Other authors classify the neuroblastomas in undifferentiated, poorly differentiated, or differentiated $(4,5)$.

Staging strategy is based on: CT or MRI scan, bone scintigraphy and histological proof by biopsy, including bone marrow biopsy (6).

\section{Assessment of the neuroblastomas by CT and MRI}

Most of the patients with supposed abdominopelvic mass are referred to a first line imaging exam, like ultrasound, while patients with suspected mediastinal mass are referred for a thoracic X-ray examination.

Subsequently, the children with lesions detected by ultrasound or thoracic X-ray, according with the clinical and biological data, with a supposed diagnosis of neuroblastoma are furthermore referred to a sectional exam like CT or MRI scan in order to evaluate the local spread or systemic involvement of the disease, before starting the treatment.

Table 1 - The International Neuroblastoma Staging System

Stage 1: localized tumor confined to the area of origin with complete gross resection or without microscopic residual disease; identifiable ipsilateral or contralateral lymph nodes negative macroscopically

Stage 2A: localized tumor with complete gross excision; identifiable ipsilateral or contralateral lymph nodes negative microscopically

Stage $2 \mathrm{~B}$ : unilateral tumor with complete or incomplete gross excision with positive ipsilateral regional lymph nodes; contralateral lymph nodes negative microscopically

Stage 3: tumor infiltrating across the midline with or without regional lymph node involvement; unilateral tumor with contralateral lymph node involvement; midline tumor with bilateral regional lymph node involvement

Stage 4: dissemination of tumor to distant lymph nodes, bone, bone marrow, liver or other organs

Stage 4S: localized primary tumor (1, 2A or $2 \mathrm{~B})$ with dissemination limited to skin, liver or bone marrow $(<10 \%$ tumor cells and MiBG scan negative in the marrow); limited to infants $<1$ year of age 


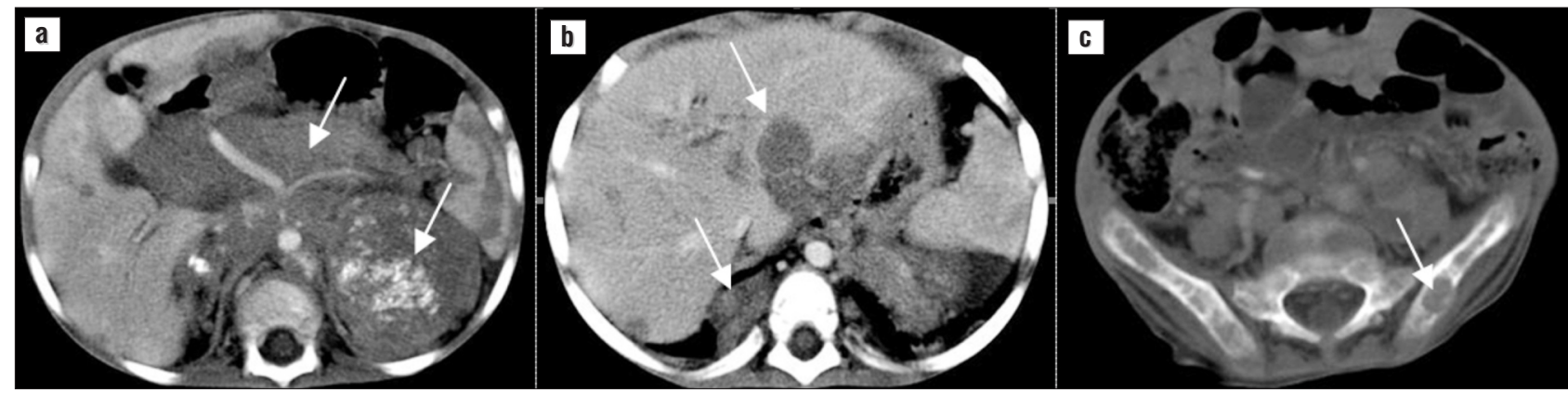

Figure 1 - Enhanced CT scan of a tree years old boy, arterial phase: multiple retroperitoneal masses with granular microcalcifications; both adrenals glandes involved; characteristic engulfing of all retroperitoneal vascular structures (a). Portal phase: hepatic and retrocrural metastases (b); enhanced CT scan, portal phase, bone window: multiple bone metastases, with cortical rupture and small parts invasion; confirmed histopatologically (c)
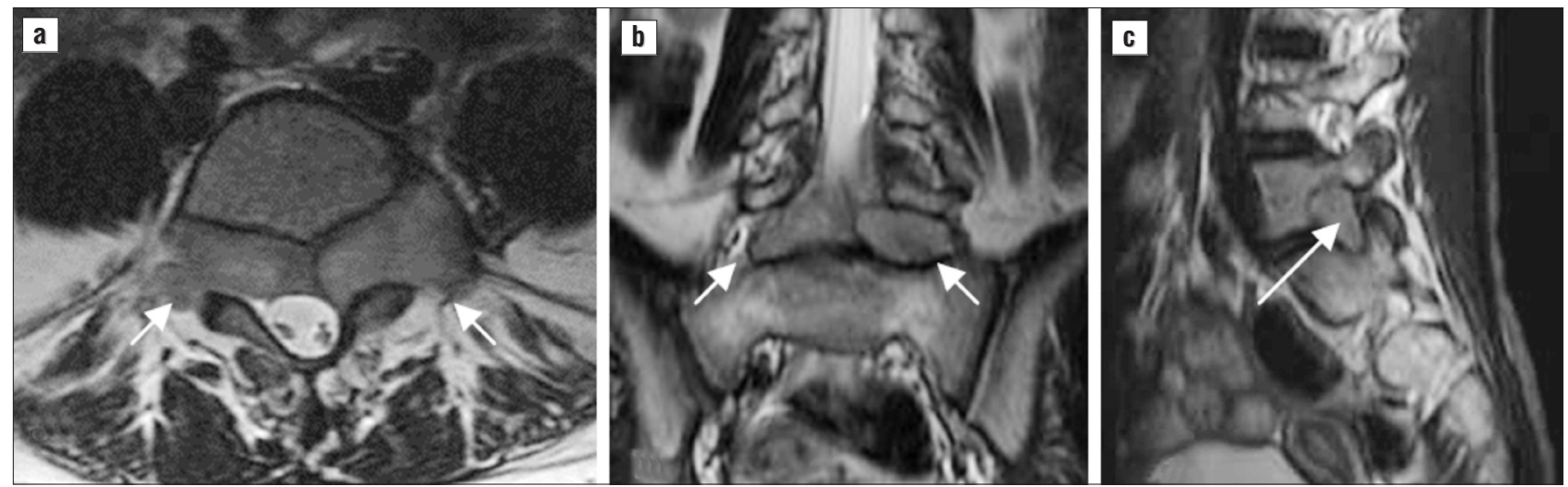

Figure 2 - 11 years old girl, non-enhanced MRI, T2wi, axial: bilateral intraforaminal masses "hourglass" typical appearance (a). Coronal and sagittal T2 weighted images of the same case, showing abnormal disappearance of the intraforaminal fat due to tumoral masses $(b, c)$

For initial staging, CT or MRI imaging are mandatory in correlation with other exams like bone scintigraphy and the histopathological confirmation of one of the neuroblastoma's histological types.

A CT scan is very efficient in detecting and staging thoracic and abdominal neuroblastoma because of the excellent ability to characterize calcified tumors and to visualize after contrast administration the vascular structures usually involved in this multiregional spread tumor (fig. 1a). CT allows also the diagnosis of organ metastases, like the liver (fig. 1b), or, in some cases, cancellous bone, especially if there are multiples and confluent lesions that destroy cortical margin and invades adjacent structures (fig. 1c).

The MRI is very useful particularly in patients with central nervous system involvement, because of its special capacity to detect peri-vertebral or peri-spinal lesions, which sometimes develop in the foraminal space (fig. 2a); MRI imaging with multiplanar acquisitions allows a precise diagnosis, visualizing foraminal masses in all the tree dimensions (fig. $2 b$ and $c$ ).
Unlike the $\mathrm{CT}, \mathrm{MRI}$ can provide all body imaging that can detect and describe multiple neuroblastoma locations, without irradiating the patient and even without contrast administration: multiple abdominal and pelvic extensive tumoral lesions and numerous peritoneal metastases with consequent large quantity of ascites (fig. $3 a$ and $b$ ). The coronal space can give global vision of the tumoral spread (fig. 3c).

The $\mathrm{CT}$ gives solid data for:

- detection of the primary site and his local spread in thoraco-abdominal and pelvic lesions;

- proper evaluation of secondary dissemination for cranial and thoraco-abdomino-pelvic regions.

The $\mathrm{CT}$ is especially useful in detection of structural microcalcifications $(79 \%$ of neuroblastomas contains calcifications) (2) and recognition and permeability evaluation of the vascular structures that are surrounded and engulfed by the tumoral nodules, like celiac artery, superior mesenteric artery, aorta, renal vessels, in which case the patient became inoperable in the premier line $(2,7)$. 

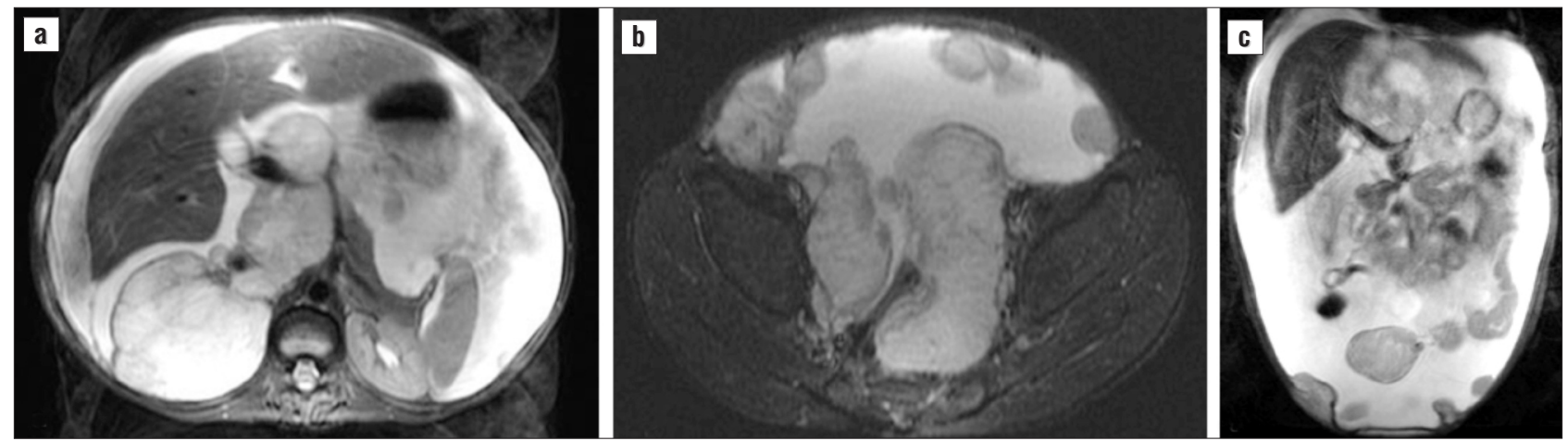

Figure 3 - Non-enhanced MRI of an 11 years old boy, axial fat sat T2wi, images: multiple retroperitoneal masses involving right adrenal gland, median retroperitoneal space, presacrat and bilateral periarterial iliac sympathetic ganglions; anterior and lateral peritoneal pelvic metastases; high signal intensity and well delineating tumors that engulf all retroperitoneal and pelvic arterial and venous structures

$(\mathrm{a}, \mathrm{b})$. Coronal non-enhanced T2wi, image showing abdominal and pelvic masses, peritoneal carcinomatosis and large ascites (c)

Abdominal CT scan can be the gold standard for detection and local development of the neuroblastoma (6).

The MRI is useful especially if:

- there are primary or secondary lesions in craniospinal and cervical regions, with a particular sensitivity in spinal extension (6), because the characteristic tendency of neuroblastoma to invade spinal canal via neuroforamina (7);

- there are pelvic primary or secondary lesions, or small parts invasion from a primary lesion;

- there are mediastinal primary tumors with typical posterior mediastinal location;

- there are bone or bone marrow metastases (4).

For thoracic (concerning lungs) or abdominal lesions, MRI is less effective than CT scan for local spread or anatomical details, because of the artifacts developed if there are patient respiratory or body movements.

Finally, the role of CT or MRI imaging is also important in establishing the prognosis: the "Children's Oncology Group" classifies neuroblastoma patients into low, intermediate and high risk categories based on the age of the patient, the stage of the disease, histological type and genetic characters of the tumor (8).

\section{CONCLUSIONS}

The $\mathrm{CT}$ and MRI imaging are mandatory diagnostic tools in staging neuroblastoma, finding the optimal moment for the surgical treatment and establish the risk categories of the children.

\section{REFERENCES}

1. Donnelly LF, O'Hara SM, Westra SJ, Blaser SI. Pocket RadiologistPediatric 100 Top Diagnoses. Amirsys. 2002:199-201.

2. Brisse $H$, Edeline V, Michon J, Couanet D, Zucker ZM, Neuenschwander S. Stratégie actuelle d'imagerie des néuroblastomes. J Radiol. 2001 Apr;82(4):447-54. French

3. Popoiu MC, David VL, Boeriu E, Popoiu AV, Arghirescu S, Vladescu $C$ et al. Nefroblastomul in Romania, unde ne situam si ce este de facut. Revista "Management in sanatate" XVI/1/2012: 27-29. Roumanian.

4. Siegel MJ, Ishwaran H, Fletcher BD, Meyer JS, Hoffer FA, Jaramillo $D$, et al. Staging of neuroblastoma at imaging: report of the radiology diagnostic oncology group. Radiology. 2002 Apr;223(1): 168-75.

5. Russell HV, Shohet JM, Nuchtern JG. Epidemiology, pathogenesis, and pathology of neuroblastoma. Available from: http://www.uptodate.com.

6. Abo-Elenain A, Naiem $\mathrm{Y}$, Hamed H, Emam M, Elkashef W, AbdelRafee A. Right adrenal gland neuroblastoma infiltrating the liver and mimicking mesenchymal hamartoma: a case report. Int $J$ Surg Case Rep. 2015;12:95-8.

7. Donnelly LF, Merrow C, Jones BV, O'Hara SM, Anton CG, Richardson RR et al. Diagnostic Imaging-Pediatrics. Second Edition, Amirsys. 2012:108-111.

8. Al-Tonbary Y, Badr M, Mansour A, El Safy U, Saeed S, Hassan T, et al. Clinico-epidemiology of neuroblastoma in north east Egypt: A 5-year multicenter study. Oncol Lett. 2015 Aug;10(2):1054-1062. 